\title{
Aerococcus urinae Endocarditis: Case Report and Review of the Literature
}

\author{
C. Ebnöther, M. Altwegg, J. Gottschalk, J.D. Seebach, A. Kronenberg
}

\begin{abstract}
Aerococcus urinae is a rare cause of urinary tract infections, mainly in elderly men with underlying urinary tract pathologies. In addition, it has been described as a pathogen in balanitis, soft tissue infections, septicemia and endocarditis. To date ten cases of $A$. urinae endocarditis have been reported in the literature with a high rate of mortality (7/10) and morbidity, as two out of three survivors suffered from neurovascular complications. Here we present the case of an additional patient who was successfully treated with surgical valve replacement and antibiotic therapy consisting of ceftriaxone and netilmicin for 6 weeks. Furthermore, we review all reported cases of $A$. urinae endocarditis with emphasis on predisposing factors and therapeutic options.

Infection 2002; 30: 310-313

DOI 10.1007/s15010-002-3106-X
\end{abstract}

\section{Introduction}

For many years the genus Aerococcus comprised the single species Aerococcus viridans, which was isolated from a broad range of habitats but rarely has been associated with human infections [1]. Aerococcus urinae was first described as an Aerococcus-like organism (ALO) by Colman et al. in 1967 [2] and was found to differ from $A$. viridans by $16 \mathrm{~s}$ rRNA sequencing [3]. A. urinae is mainly responsible for urinary tract infections, where it accounts for $0.3-0.8 \%$ of all cases $[4,5]$. A. urinae is also a pathogen reported in balanitis [6], soft tissue infection [6], empyema [2], bacteremia and endocarditis [5, 7,8]. Between 1987 and 1994 the overall incidence of bacteremia associated with ALO in Denmark was 0.5 cases per million inhabitants per year [7]. Here we present the first case of an $A$. urinae endocarditis diagnosed by broad-spectrum polymerase chain reaction (PCR) of the resected valve specimen.

\section{Case Report}

A 75-year-old man was admitted to our tertiary care hospital with blood culture negative aortic valve endocarditis for valve replacement. Three months earlier he had been treated as an out- patient with ciprofloxacin for dipstick-positive urinary tract infection for 7 days; urine culture was not done at this time. After 1 week a urinary retention due to urethrastenosis and phimosis was treated with transabdominal catheterization. The patient was prescribed ciprofloxacin prophylactically. A urine specimen did not show pyuria and urine cultures again were not performed. 50 days before admission to our hospital, urinary tract obstruction was corrected surgically and ciprofloxacin was prescribed again prophylactically; the catheter was removed after 10 days and ciprofloxacin was stopped. The patient remained asymptomatic for 20 days before he suddenly suffered from chills and fever up to $39^{\circ} \mathrm{C}$. He immediately was admitted to the urologic department of another hospital (20 days prior to admission to our hospital) and was treated with ciprofloxacin and netilmicin empirically on suspicion of a urinary tract infection, although there was no pyuria. Urine cultures were obtained only after start of the antibiotic therapy and remained negative, blood cultures were not done. After initial defervescence he became febrile again 7 days later and ciprofloxacin/netilmicin were replaced by meropenem without improvement.

Five days later a new systolic-diastolic murmur was noted at the left sternal border and an indolent, blue to black flat lesion of about $2 \mathrm{~mm}$ diameter was detected paraunguinally on the right fifth toe. The laboratory values were as follows: hemoglobin $10.2 \mathrm{~g} / \mathrm{dl}$, leukocytes $9.3 \times 10^{9} / 1$, thrombocytes $266 \times 10^{9} / 1$, C-reactive protein (CRP) $180 \mu \mathrm{g} / \mathrm{l}$ and creatinine $118 \mathrm{mmol} / \mathrm{l}$. The urine sediment was normal, blood and urine cultures were negative as well. Transesophageal echocardiography showed vegetations on the aortic valve (Figure 1), CT of the abdomen a left kidney infarction and magnetic resonance imaging of the cerebrum, performed because of hallucinations, multiple ischemic lesions. A diagnosis of infective aortic valve endocarditis with systemic embolization was made. The causative microorganism, however, remained obscure and antibiotic therapy was changed to flucloxacillin, penicillin and netilmicin. Despite this therapy, the pa-

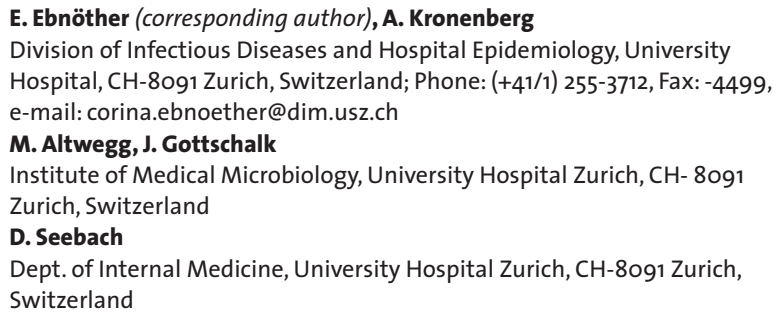

Received: June 24, $2002 \cdot$ Revision accepted: September 4, 2002 


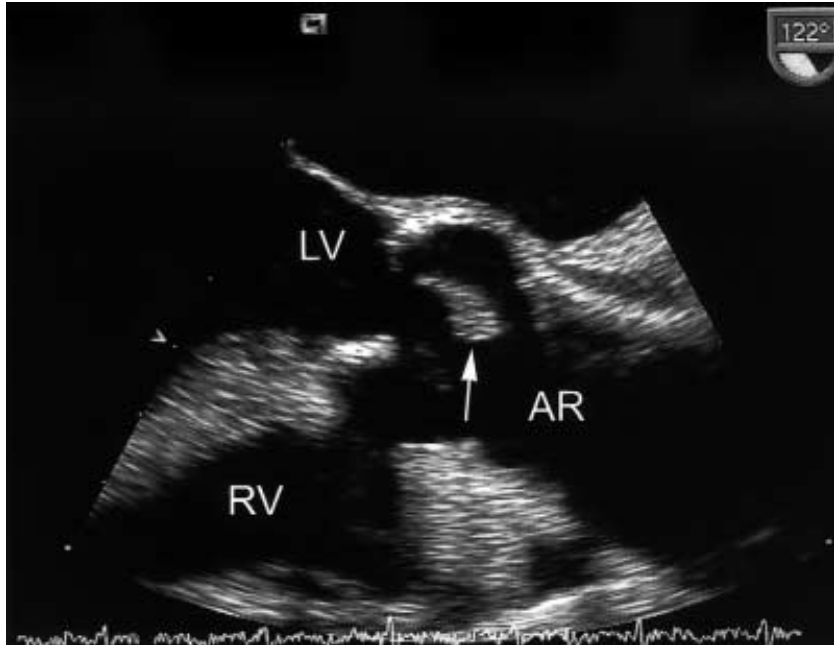

Figure 1. Transesophageal echocardiography visualizes the vegetation on the aortic valve (white arrow). LV: left ventricle; RV: right ventricle; AR: aortic root.

tient remained febrile. Echocardiography was repeated 1 week later and showed progressive destruction of the aortic valve, whereupon the patient was transferred to our hospital.

At the day of admission to our hospital the antibiotic regimen was modified as follows: netilmicin was replaced by gentamicin, flucloxacillin $4 \times 2 \mathrm{~g}$ iv was maintained, on suspicion of brain abscesses, penicillin was replaced by ceftriaxone $2 \times 2 \mathrm{~g}$ iv and ciprofloxacin $2 \times 750 \mathrm{mg}$ po was added until the negative serologic results for Coxiella burnetii, Bartonella spp. and Brucella melitensis were available. With this therapy the patient became afebrile and the CRP level dropped from 141 to $69 \mathrm{mg} / \mathrm{l}$ at day 8 after hospitalization. Because of progressive aortic insufficiency valve replacement was performed 12 days after admission to our clinic. A Gram stain of the valve specimen obtained from the operating room showed predominantly gram-positive cocci with morphologic variations, explainable by ongoing antibiotic therapy. However, cultures of the valve remained negative, while amplification and direct sequencing performed on DNA isolated from valve material, as described by Goldenberger et al. [9], resulted in a 374 bp fragment that perfectly matched an $A$. urinae sequence (accession number U64459) [9]. The next most closely related species was $A$. urinaehominis with 18 mismatches in a stretch of 350 nucleotides. Upon this result, antibiotic therapy was simplified to ceftriaxone $2 \times 2 \mathrm{~g}$ iv and netilmicin iv guided by drug levels which were continued for 6 weeks postoperatively. The further course of the patient was unremarkable; 4 months later he was doing well with a good prosthetic valve function and without any neurologic sequelae.

\section{Discussion}

A. urinae is a gram-positive, microaerophilic, catalase-negative, alpha-hemolytic coccus, growing predominately in tetrads and clusters. In contrast to streptococci, it is capable of growing in $6.5 \% \mathrm{NaCl}$. Its growth on $5 \%$ sheep-blood agar depends on incubation in $\mathrm{CO}_{2}$, therefore growth on urine-dipslide agar is not reliable. This might explain discrepancies between positive blood and negative urine cultures in some cases [7]. Further underdiagnosis is possible, as the database of the API 20 Strep system (Version 6.9;
API bioMérieux, Marcy l'Etoile, France), which has been evaluated for identifiaction of streptococci associated with infective endocarditis but does not contain $A$. urinae in the database. This system may identify $A$. urinae as Streptococcus acidominus with a high diagnostic likelyhood, but low $\mathrm{T}$ values (which measure closeness to ideal phenotype of a species). This constellation must raise the suspicion of a wrong identification and mandates conventional testing or molecular sequencing [10]. Differentiation from $A$. viridans relies on positive reactions for leucine arylamidase and beta-glucoronidase [3]. Besides $A$. viridans and $A$. urinae, three additional Aerococcus species have been recently isolated from the human vagina, Aerococcus christensenii [11], and from human urine, A. urinaehominis and Aerococcus sanguicola, respectively $[12,13]$. However, the pathogenic importance of these latter organisms remains unknown.

A total of eleven cases (including our patient) of $A$. urinae endocarditis have been reported in the literature to date (Table 1). Our patient presented with the typical predisposing factors for $A$. urinae endocarditis which are male sex $(9 / 11)$, age $>65(8 / 11)$ and preexisting urinary tract pathologies $(5 / 11)$, such as prostata hyperplasia, urethral stricture and prior urinary tract surgery. The latter reflects that the urinary tract is a potential port of entry for this organism. Indeed, 6/11 patients were empirically treated for symptomatic urinary tract infections before diagnosis of $A$. urinae endocarditis. Two frequently used antibiotics for urinary tract infections, cotrimoxazole and quinolones, have no or only moderate activity against $A$. urinae. Therefore, it is probable that $A$. urinae may be selected during empiric therapy with these drugs. As with all cases of infective endocarditis, preexisting valvular dysfunction is another risk factor, which was described in 3/11 patients, but was not documented in our case, although unrecognized sclerotic aortic valves are common in this age-group. Remarkably, these three patients had valvular pathologies with only moderate risk for endocarditis, stressing the importance of other predisposing factors. Finally, systemic comorbidities such as ischemic heart disease, diabetes mellitus and malignancy have been proposed as risk factors for $A$. urinae endocarditis [4, 5,8 ] and were present in 4/11 patients; this may, however, just be due to the median age in this patient group.

Although its risk factor profile is quite characteristic, A. urinae endocarditis is clinically indistinguishable from endocarditis caused by other bacterial agents. It presents with fever (9/11), a new heart murmur (7/11) and vegetations (8/11) (data not shown). Blood cultures are positive in $10 / 11$ patients. Occuring in $55 \%$ of the patients, systemic embolization seems to be frequent, compared to only 20$40 \%$ in infective endocarditis cases caused by other microorganisms [14]. This might be due to late diagnosis because of the subacute character of the disease and the often unspecific symptoms in the elderly. The establishment of the etiologic diagnosis relied on positive blood cultures in all but our patient, where blood cultures were performed only after start of antibiotic treatment and remained nega- 


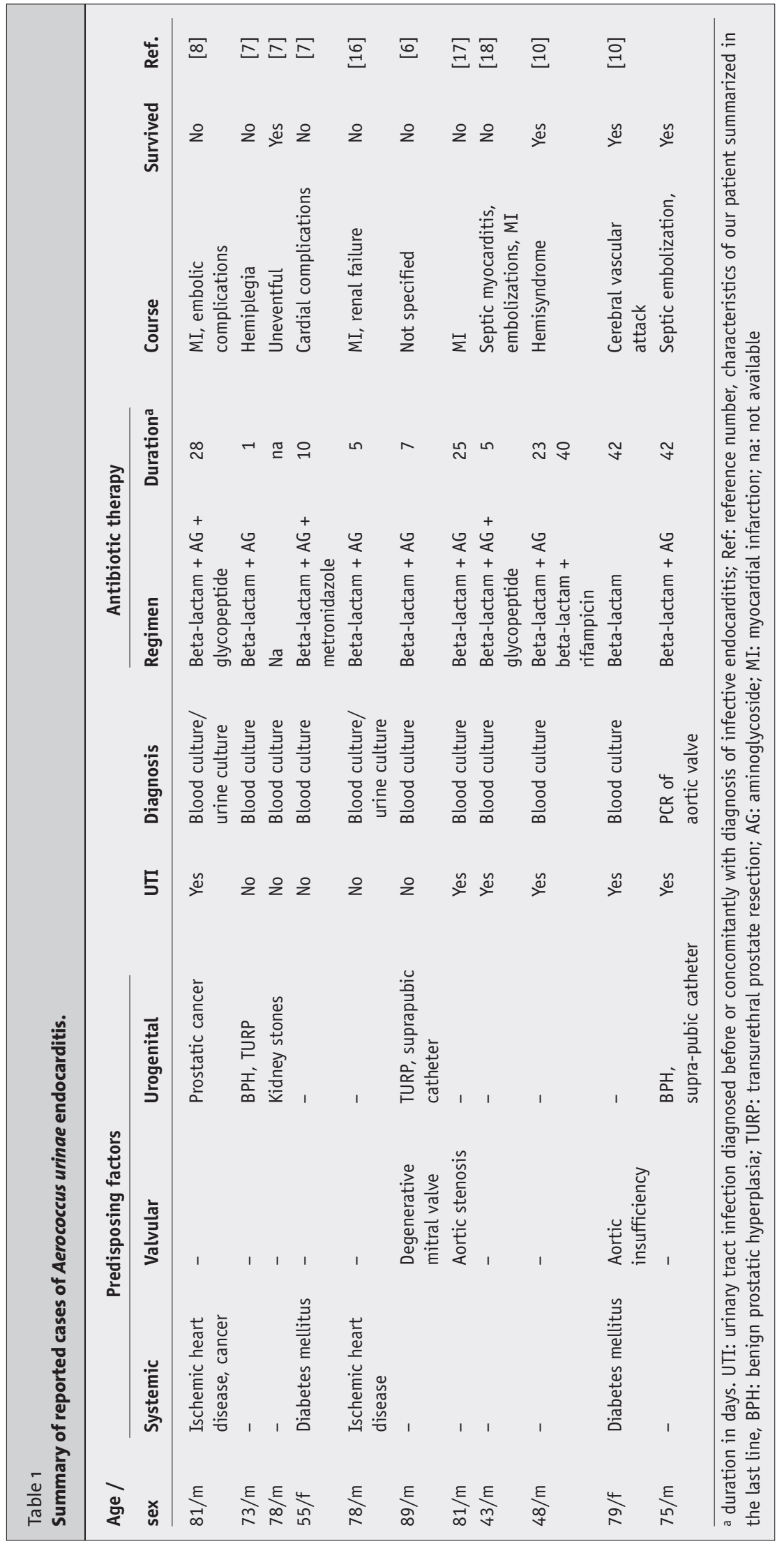

tive. To the best of our knowledge this is the first case of $A$. urinae endocarditis where broad-spectrum PCR from a surgically removed aortic valve led to identification of the organism. In the future this sensitive and specific method could help identify even more cases of A. urinae endocarditis, which may have been missed so far because of negative blood cultures.

Due to the rareness of $A$. urinae endocarditis, controlled studies are not possible and therefore antibiotic treatment is not standardized. In vitro susceptibilities of 56 isolates showed little inter-isolate variability with low minimal inhibitory concentrations (MIC) for penicillin, amoxicillin, piperacillin, cefepime, vancomycin and rifampicin, but variable MIC for ceftriaxone. No isolate showed high-level aminoglycoside resistance [15]. Furthermore, synergy testing using time-killing curves showed a synergistic bactericidal activity when penicillin or vancomycin were combined with gentamicin $[10,15]$.

Treatment modalities were available for 10/11 patients. Although nine of these patients were treated with a combination of a beta-lactam and an aminoglycoside, seven of them died. The remaining patient was treated with a betalactam alone and survived with neurologic sequelae. In all lethal cases death occured during the first 4 weeks (mean 12 days). The optimal duration of therapy for A. urinae endocarditis remains to be defined. Nevertheless, we suggest to treat all patients intravenously for a minimum of 6 weeks, since all surviving patients, including the presented patient, were treated for this time period either with a beta-lactam alone or with a beta-lactam and an aminoglycoside. Surgical valve replacement does not seem to be a prerequisite for cure, as our patient was the first to undergo this treatment.

As mentioned above, the prognosis of $A$. urinae endocarditis is poor. Moreover, even if patients survive an A. urinae endocarditis, morbidity is high since 3/4 survivors, including our patient, suffered from neurologic sequelae after cerebrovascular embolization. We suppose that late diagnosis because of the 
oligosymptomatic clinical presentation in elderly patients, as well as comorbidities, contribute essentially to the observed high early mortality and embolization rate.

In summary, $A$. urinae endocarditis is a severe disease affecting mainly elderly men with urinary tract pathologies and comorbidities. We suppose that late diagnosis due to unspecific symptoms in the elderly and the difficulty to cultivate the organism, as well as comorbidities, contribute to the high morbidity and mortality. Antibiotic therapy with beta-lactams for at least 6 weeks is effective if patients survive the 1 st weeks of therapy. The addition of aminoglycosides probably has a synergistic effect.

\section{References}

1. Facklam R, Elliott JA: Identification, classification, and clinical relevance of catalase-negative, gram-positive cocci, excluding the streptococci and enterococci. Clin Microbiol Rev 1995; 8: 479-495.

2. Colman G: Aerococcus-like organisms isolated from human infections. J Clin Pathol 1967; 20: 294-297.

3. Aguirre M, Collins MD: Phylogenetic analysis of some Aerococcus-like organisms from urinary tract infections: description of Aerococcus urinae sp. nov. J Gen Microbiol 1992; 138: 401-405.

4. Schuur PM, Kasteren ME, Sabbe L, Vos MC, Janssens MM, Buiting AG: Urinary tract infections with Aerococcus urinae in the south of The Netherlands. Eur J Clin Microbiol Infect Dis 1997; 16: 871-875.

5. Christensen JJ, Korner B, Kjaergaard H: Aerococcus-like organism - an unnoticed urinary tract pathogen. APMIS 1989; 97: 539-546.

6. Schuur PM, Sabbe L, van der Wouw AJ, Montagne GJ, Buiting AG: Three cases of serious infection caused by Aerococcus urinae. Eur J Clin Microbiol Infect Dis 1999; 18:368-371.

7. Christensen JJ, Jensen IP, Faerk J, Kristensen B, Skov R, Korner B: Bacteremia/septicemia due to Aerococcus-like organisms: report of seventeen cases. Danish ALO Study Group. Clin Infect Dis 1995; 21: 943-947.

8. Christensen JJ, Gutschik E, Friis-Moller A, Korner B: Urosepticemia and fatal endocarditis caused by Aerococcus-like organisms. Scand J Infect Dis 1991; 23: 717-721.

9. Goldenberger D, Kunzli A, Vogt P, Zbinden R, Altwegg M: Molecular diagnosis of bacterial endocarditis by broad-range PCR amplification and direct sequencing. J Clin Microbiol 1997; 35: 2733-2739.

10. Zbinden R, Santanam P, Hunziker L, Leuzinger B, von Graevenitz A: Endocarditis due to Aerococcus urinae: diagnostic tests, fatty acid composition and killing kinetics. Infection 1999; 27: 122-124.

11. Collins MD, Jovita MR, Hutson RA, Ohlen M, Falsen E: Aerococcus christensenii sp. nov., from the human vagina. Int J Syst Bacteriol 1999; 49: 1125-1128.

12. Lawson PA, Falsen E, Ohlen M, Collins MD: Aerococcus urinaehominis sp. nov., isolated from human urine. Int J Syst Evol Microbiol 2001; 51: 683-686.

13. Lawson PA, Falsen E, Truberg-Jensen K, Collins MD: Aerococcus sanguicola sp. nov., isolated from a human clinical source. Int J Syst Evol Microbiol 2001; 51: 475-479.

14. Dajani AS, Taubert KA, Wilson W, Bolger AF, Bayer A, Ferrieri P, Gewitz MH, Shulman ST, Nouri S, Newburger JW, Hutto C, Pallasch TJ, Gage TW, Levison ME, Peter G, Zuccaro G Jr.: Prevention of bacterial endocarditis: recommendations by the American Heart Association. Clin Infect Dis 1997; 25: 1448-1458.

15. Skov R, Christensen JJ, Korner B, Frimodt-Moller N, Espersen F: In vitro antimicrobial susceptibility of Aerococcus urinae to 14 antibiotics, and time-kill curves for penicillin, gentamicin and vancomycin. J Antimicrob Chemother 2001; 48: 653-658.

16. Kristensen B, Nielsen G: Endocarditis caused by Aerococcus urinae, a newly recognized pathogen. Eur J Clin Microbiol Infect Dis 1995; 14: 49-51.

17. Skov RL, Klarlund M, Thorsen S: Fatal endocarditis due to Aerococcus urinae. Diagn Microbiol Infect Dis 1995; 21: 219-221.

18. Gritsch W, Nagl M, Hausdorfer J, Gschwendtner A, Pechlaner C, Wiedermann CJ: Septicaemia and endomyocarditis caused by Aerococcus urinae. Wien Klin Wochenschr 1999; 111: 446-447. 